\title{
Stem Cells Enhance Tissue Engineering in Head and Neck Surgery
}

\author{
Rwik Sen, Ph.D* \\ Active Motif Inc, Carlsbad, California, USA
}

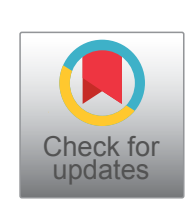

\begin{abstract}
Stem cells have shown therapeutic promise against various diseases. Recent advances in tissue engineering show that applications of stem cells significantly improve the tissue regeneration process. In this direction, studies have shown the promise of stem cells in head and neck surgery to treat various associated diseases. The inherent properties of stem cells like self-renewal and maintenance of pluripotency aid the process of quicker healing and tissue regeneration in head and neck diseases. Although the field is new and several avenues remain to be explored, the promise shown by stem cells so far are sufficient to continue expanding their applications for various therapeutic applications. Stem cells are administered in combination with biomaterials and therapeutic molecules repair damaged tissue. Surgery of the head and neck including ear, nose, throat, eye, burns, scalp and skin grafting have all been benefitted from stem cells. This mini-review presents the potential of stem cells in the context of head and neck surgery.
\end{abstract}

\section{Introduction}

According to the American Society for Clinical Oncology, head and neck cancers constitute about $4 \%$ of all cancers in the United States, where, an estimated 65,630 people will be afflicted with head and neck cancer, with an estimated 14,50 deaths in one year. Several factors cause these cancers genetic and epigenetic mutations, human papilloma virus, and environmental reasons like tobacco and alcohol. One of the major interventions against head and neck cancer is surgery with reconstructive therapy [1]. However, several limitations exist with traditional surgical processes where the recent applications of stem cells have shown considerable promise.

Head and neck cancer comprises of $4 \%$ of all cancers in the United States, affecting over 65,000 people and killing close to 15,000 people in one year. Stem cells have shown promise in head and neck cancers as reported by Cohen, et al. [2] where they labeled gold nanoparticles with exosomes derived from mesenchymal stem cells [2]. Upon intravenous injection, mesenchymal stem cells-derived exosomes exhibited very high tumor homing ability, penetration and accumulation in the bulk and cytoplasm of tumors [2]. Hence, for head and neck surgery and therapies against cancers of those regions, stem cells show extremely promising qualities for tumor-targeted therapy.

Head and neck region is complex and consists of several crucial organs for which stem cells have shown promising repair and regeneration potential. For organs like eyes, stem cell-based therapies and surgeries have opened new avenues. Retinal, corneal, and macular damage, as well as glaucoma, are some areas where the potential of stem cells are explored
[3-8]. For cornea, fetal cartilage-derived stem cells differentiated into sheets of epithelial cells that expressed corneal epithelial cell markers. The differentiated cells repaired damaged cornea [5,6]. Retinal pigment epithelial cells derived from human embryonic and induced pluripotent stem cells have been used for cell transplantation in clinical research of age-associated macular degeneration [7]. The cells are chosen because of their unique inflammatory characteristics like immunosuppression and immunogenicity [7].

Although the use of stem cells in head and neck surgery are at very initial stages at present, further development of this technology is bound to significantly improve the quality of life of patients undergoing head and neck surgery. In this direction, this mini-review highlights the benefits and potential of stem cells in head and neck surgery.

\section{Stem Cells Show Therapeutic Promise against Head and Neck Diseases}

Reconstructive head and neck surgery is a very complicated undertaking because tissues in those regions are derived from multiple cell types during craniofacial development. Further, there is a requirement of follow-up therapy after the

*Corresponding author: Dr. Rwik Sen, Active Motif Inc, 58187, 1914 Palomar Oaks Way, Suite 150, Carlsbad, California, USA

Accepted: February 13, 2021

Published online: February 15, 2021

Citation: Sen R (2021) Stem Cells Enhance Tissue Engineering in Head and Neck Surgery. J Head Neck Surg 3(1):133-136 
initial treatment to prevent secondary tumor formation. Additionally, there is a dearth of source tissue donors as well as the issue of recipient's immune system adversely reacting to the implant. Stem cell therapy in head and neck surgery shows promise in the face of the above adversities because stem cells have the capacity to self-renew and maintain pluripotent state [9]. The most important organ of the head and neck region is the brain which has also greatly benefitted from stem cell therapy. During traumatic brain injury injured brain tissue is repaired by exogenous stem cells which migrate to the damage site, differentiation to repopulate the site with healthy new cells, secrete anti-inflammation and growth factors, and improve neurological function [10]. Administration of molecules like cur cumin further enhances the process [11]. Stem cell therapy has also benefitted nasal diseases like polyposis [12], ear disorders and inner ear or ganoids development $[13,14]$. The benefits of stem cells to eyes and throat are described in the sections below. Hence, several studies have reported the potential of stem cells against diseases and in engineering of tissues like nerve, vessels, cartilage, bones, trachea, and esophagus [15-17].

\section{Stem cells in Tissue Engineering}

For successful tissue engineering, biomimetic scaffolds are seeded with cells which populate the region and regenerate the tissue. The scaffold provides extracellular support for the cells, and growth factors are provided to the cells for their propagation. Based on this technology, stem cells have been used to engineer several tissues including those of the head and neck region as discussed below (Figure 1).

\section{Cartilage}

Several methodologies are adopted for the promise of mesenchymal stem cells (MSCs) in the repair of cartilage [18]. As dermis isolated adult stem cells (DIAS), MSCs are capable of differentiating to several lineages including chondrogenic. MSCs cultured in chondrogenic media leads to upregulation of genes associated with cartilage development like collagen type II and COMP [18]. DIAS treated with transforming growth factor $\beta 1$ (TGF- $\beta 1$ ) or bone morphogenetic protein 2 (BMP-2) elevates sulfated glycosaminoglycan which is structural component of cartilage. Liposome-mediated TGF- $\beta 1$ transfer into MSCs on chitosan scaffolds resulted in genetically engineered cartilage that was implanted to repair articular cartilage defects [19]. MSCs derived from bone marrow have been seeded on decellularized donor trachea to engineer trachea for human transplantation because the cells differentiate into a chondrocyte lineage [20]. MSCs derived from adipose tissue enhances tissue regeneration and survival of scaffolds that are bioengineered to replace trachea [21].

\section{Bone}

Stem cells have shown promise in engineering of bones, including those found in the head and neck [22]. In a study to augment sinus floor or increasing bone in the upper jaw, more bone formation was observed when scaffold combined with autologous BM (bone marrow) MSCs were provided to patients compared to autologous bone graft [23]. Cultured autologous BM MSCs applied on hydroxyapatite - $\beta$ tricalcium phosphate ceramic successfully elevated the maxillary sinus [24]. Cleft-lip and cleft-palate are craniofacial defects which are the most common birth defects according to Centers for Disease Control and Prevention (CDC), USA. MSCs seeded on demineralized bone matrix with calcium sulphate show promise in reconstructing alveolar defects in patients having unilateral cleft palates [25]. In another methodology, MSCs can be cultured in osteogenic media on scaffolds to develop bones which are then implanted as a treatment for jaw bone defects [26].

\section{Vocal folds}

Studies have shown that MSCs incorporated into a HA hydrogel construct hold therapeutic promise against vocal fold scarring caused by disease or procedures like partial laryngectomy. This is achieved by enhanced wound healing through the secretion of bioactive molecules and restoration of the dimensions of the superficial lamina propria or connective tissue lining of tubes like respiratory tract [22]. BM MSCs

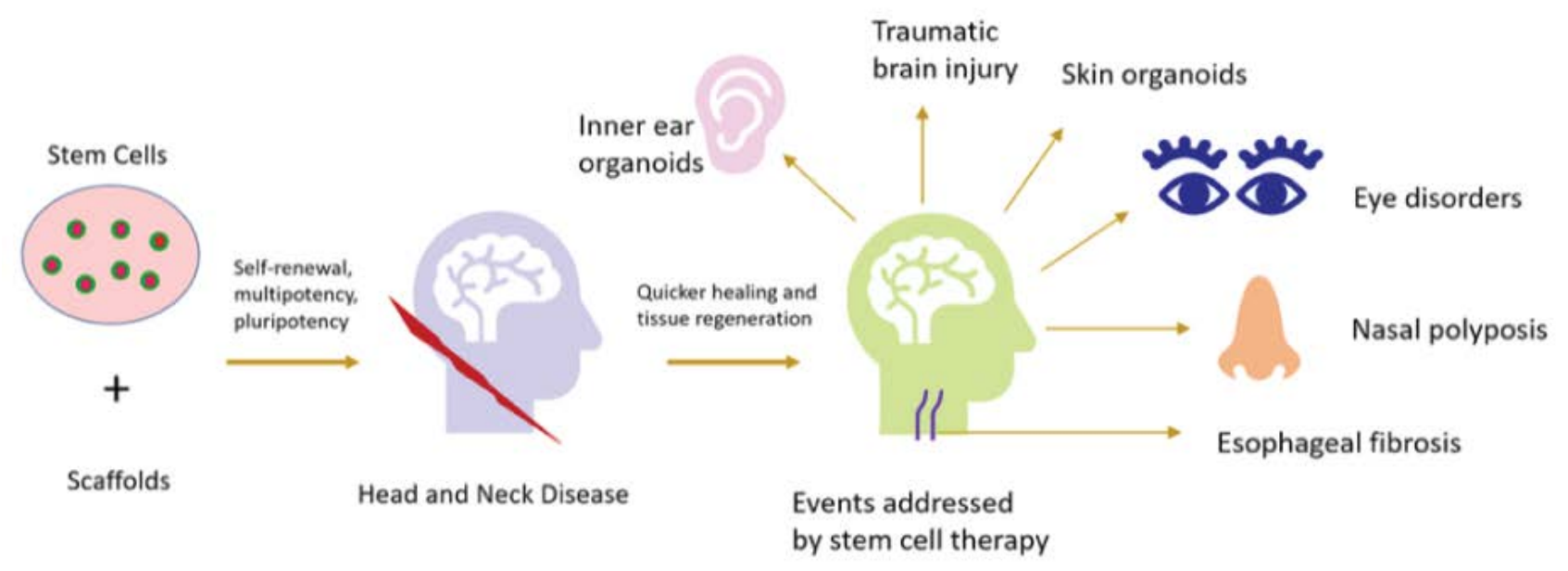

Figure 1: Stem cells applications and therapies improve patient outcomes in head and neck disease. 
that are autologously implanted in vocal fold are found to be propagating with the expressions of epithelial tissue and muscle markers indicating differentiation [27].

\section{Retina}

Several studies have shown that administration of stem cells in retinal degeneration or injury conditions have led to significant improvement and regeneration. In one study, a combination of sub retinal transplantation and intravenous injection of human dental pulp stem cells were performed to recover retinal degeneration [28]. The therapy led to improved retinal outer nuclear layer thickness structurally, and improvement in photoreceptors or cone cells functionally [28]. Stem cells from bone marrow mononuclear fraction have been applied at intravitreous sites in Stargardt dystrophy patients leading to improvements in best corrected visual acuity and microperimetry [3]. The observations prove the safety and effectiveness of stem cells invasive therapies using stem cells in eye disease.

\section{Skin}

Pluripotent stem cells can be used to develop skin or ganoids for various patients which are appendage-bearing skin tissue [29]. Stem cells have greatly helped in overcoming traditional methods of skin cells culturing because cultures do not recapitulate the complexity found in native skin [29]. For example, skin appendages like sweat glands and hair follicles are very difficult to generate, maintain in cultures, and reconstruct during skin damage. Another aspect of therapy and surgery based on skin is needed during burns and wound healing [30]. Stem cells show potential to heal burn wounds, like hypertrophic scars, for the processes of inflammation, proliferation and maturation [30].

\section{Conclusion}

The studies discussed above emphasize on the need for further research to optimize the applications of stem cells in head and neck therapeutics [31]. It is interesting to note that stem cells are also connected to the pathophysiology of head and neck cancers. MSCs are seen to interact with tumor cells of head and neck squamous cell carcinoma (HNSCC), and the interaction is regulated by normoxia and hypoxia [32]. Increase in reactive oxygen species (ROS) causes esophageal fibrosis upon prolonged irradiation during head and neck cancers [33] which is shown to be ameliorated by spheroids of human mesenchymal stem cells that are encapsulated in hyaluronic acid hydrogel [33]. Further research is needed to understand if the interaction can be harnessed to employ MSCs as therapeutics against HNSCC. Nonetheless, the potential of stem cells in tissue engineering bring them to the forefront as therapeutic candidates against head and neck disease. Use of stem cells in head and neck therapeutics and surgery, in combination with small molecules, biomaterials [34], organoid development, etc. have indeed improved patient outcomes. Collectively, the studies project the importance of stem cells in future head and neck therapies, including skull base surgeries [35]. With time, the full potential of stem cells will be harnessed against head and neck diseases with better outcome and improved quality of life.

\section{Conflicts of Interest}

None.

\section{References}

1. Barczak W, Golusinski P, Luczewski L, et al. (2016) The importance of stem cell engineering in head and neck oncology. Biotechnol Lett 38: 1665-1672.

2. Cohen O, Betzer O, Elmaliach-Pnini N, et al. (2021) 'Golden' exosomes as delivery vehicles to target tumors and overcome intratumoral barriers: In vivo tracking in a model for head and neck cancer. Biomater Sci.

3. Cotrim CC, Vieira Messias AM, Jorge R, et al. (2020) Intravitreal use of a bone marrow mononuclear fraction (BMMF) containing CD34+ cells in patients with stargardt type macular dystrophy. Stem Cells Int 8828256.

4. Nguyen T, Urrutia-Cabrera D, Liou RH, et al. (2020) New technologies to study functional genomics of age-related macular degeneration. Front Cell Dev Biol 8: 604220.

5. Park IS, Kim BK, Truong MD, et al. (2021) Corneal repair with adhesive cell sheets of fetal cartilage-derived stem cells. Tissue Eng Regen Med.

6. Shah R, Amador C, Tormanen K, et al. (2021) Systemic diseases and the cornea. Experimental Eye Research 204: 108455.

7. Sugita S, Mandai M, Kamao H, et al. (2021) Immunological aspects of RPE cell transplantation. Prog Retin Eye Res 100950.

8. Voicu L, Salim S (2021) New strategies for the management of ocular surface disease in glaucoma patients. Curr Opin Ophthalmol 32: 134-140.

9. Murry CE, Keller G (2008) Differentiation of embryonic stem cells to clinically relevant populations: Lessons from embryonic development. Cell 132: 661-680.

10. Zhou Y, Shao A, Xu W, et al. (2019) Advance of stem cell treatment for traumatic brain injury. Front Cell Neurosci 13: 301.

11. Attari F, Ghadiri T, Hashemi M (2020) Combination of curcumin with autologous transplantation of adult neural stem/progenitor cells leads to more efficient repair of damaged cerebral tissue of rat. Exp Physiol 105: 1610-1622.

12. Vanderhaegen T, Gengler I, Dendooven A, et al. (2021) Eosinophils in the Field of Nasal Polyposis: Towards a better understanding of biologic therapies. Clin Rev Allergy Immunol.

13. Nourbakhsh A, Colbert BM, Nisenbaum E, et al. (2021) Stem cells and gene therapy in progressive hearing loss: The state of the art. J Assoc Res Otolaryngol.

14. Van der Valk WH, Steinhart MR, Zhang J, et al. (2021) Building inner ears: Recent advances and future challenges for in vitro organoid systems. Cell Death Differ 28: 24-34.

15. Carpenter MK, Frey-Vasconcells J, Rao MS (2009) Developing safe therapies from human pluripotent stem cells. Nat Biotechnol 27: 606-613.

16. Hwang NS, Varghese S, Lee HJ, et al. (2008) In vivo commitment and functional tissue regeneration using human embryonic stem cell-derived mesenchymal cells. Proc Natl Acad Sci USA 105: 20641-20646.

17. Nishikawa S, Goldstein RA, Nierras CR (2008) The promise of human induced pluripotent stem cells for research and therapy. Nat Rev Mol Cell Biol 9: 725-729. 
18. Csaki C, Schneider PR, Shakibaei M (2008) Mesenchymal stem cells as a potential pool for cartilage tissue engineering. Ann Anat 190: 395-412.

19. Guo CA, Liu XG, Huo JZ, et al. (2007) Novel gene-modified-tissue engineering of cartilage using stable transforming growth factor-beta1-transfected mesenchymal stem cells grown on chitosan scaffolds. J Biosci Bioeng 103: 547-556.

20. Macchiarini $P$, Jungebluth $P$, Go T, et al. (2008) Clinical transplantation of a tissue-engineered airway. Lancet 372: 2023-2030.

21. Suzuki T, Kobayashi K, Tada Y, et al. (2008) Regeneration of the trachea using a bioengineered scaffold with adipose-derived stem cells. Ann Otol Rhinol Laryngol 117: 453-463.

22. King SN, Hanson SE, Hematti P, et al. (2012) Current applications of mesenchymal stem cells for tissue replacement in otolaryngology-head and neck surgery. Am J Stem Cells 1: 225-238.

23. Rickert D, Sauerbier S, Nagursky H, et al. (2011) Maxillary sinus floor elevation with bovine bone mineral combined with either autogenous bone or autogenous stem cells: A prospective randomized clinical trial. Clin Oral Implants Res 22: 251-258.

24. Shayesteh YS, Khojasteh A, Soleimani M, et al. (2008) Sinus augmentation using human mesenchymal stem cells loaded into a beta-tricalcium phosphate/hydroxyapatite scaffold. Oral Surg Oral Med Oral Pathol Oral Radiol Endod 106: 203-209.

25. Behnia H, Khojasteh A, Soleimani M, et al. (2009) Secondary repair of alveolar clefts using human mesenchymal stem cells. Oral Surg Oral Med Oral Pathol Oral Radiol Endod 108: e1-e6.

26. Meijer GJ, de Bruijn JD, Koole R, et al. (2008) Cell based bone tissue engineering in jaw defects. Biomaterials 29: 3053-3061.
27. Kanemaru S, Nakamura T, Yamashita M, et al. (2005) Destiny of autologous bone marrow-derived stromal cells implanted in the vocal fold. Ann Otol Rhinol Laryngol 114: 907-912.

28. Lam C, Alsaeedi HA, Koh AE, et al. (2021) Human dental pulp stem cells (DPSCs) therapy in rescuing photoreceptors and establishing a sodium iodate-induced retinal degeneration rat model. Tissue Eng Regen Med 18:143-154.

29. Lee J, Koehler KR (2021) Skin organoids: A new human model for developmental and translational research. Exp Dermatol.

30. Coma M, Frohlichova L, Urban L, et al. (2021) Molecular changes underlying hypertrophic scarring following burns involve specific deregulations at all wound healing stages (inflammation, proliferation and maturation). Int J Mol Sci 22: 897.

31. Sun JJ (2005) Studies of stem cells in otolaryngology-head-neck surgery. Zhonghua Yi Xue Za Zhi, 85: 2538-2540.

32. Wilhelm C, Scherzad A, Bregenzer M, et al. (2020) Interaction of head and neck squamous cell carcinoma cells and mesenchymal stem cells under hypoxia and normoxia. Oncol Lett 20: 229.

33. Kim IG, Cho H, Shin J, et al. (2021) Regeneration of irradiation-damaged esophagus by local delivery of mesenchymal stem-cell spheroids encapsulated in a hyaluronic-acid-based hydrogel. Biomater Sci.

34. Lacalle-Aurioles M, Cassel de Camps C, Zorca CE, et al. (2020) Applying hiPSCs and biomaterials towards an understanding and treatment of traumatic brain injury. Front Cell Neurosci 14: 594304.

35. Sekhar LN, Juric-Sekhar G, Qazi Z, et al. (2020) The future of skull base surgery: A view through tinted glasses. World Neurosurg, 142: $29-42$.

DOI: $10.36959 / 605 / 555$ 